\title{
TREINAMENTO MENTAL NO TÊNIS: REVISÃO SISTEMÁTICA DA LITERATURA
}

\author{
ESP. JONAS GODTSFRIEDT \\ Programa de Pós-graduação em Educação Física, \\ Centro de Desportos, Universidade Federal de Santa Catarina \\ (Florianópolis - Santa Catarina - Brasil) \\ E-mail: jog1000@hotmail.com

\section{DR. ALEXANDRO ANDRADE} \\ Centro de Ciências da Saúde e do Esporte, \\ Universidade do Estado de Santa Catarina \\ (Florianópolis - Santa Catarina - Brasil) \\ E-mail: d2aa@udesc.br
}

\section{MS. DIEGO ITIBERE CUNHA VASCONCELLOS \\ Programa de Pós-graduação em Ciências do Movimento Humano, \\ Centro de Ciências da Saúde e do Esporte, \\ Universidade do Estado de Santa Catarina \\ (Florianópolis - Santa Catarina - Brasil) \\ E-mail: diegoitibere@gmail.com}

\begin{abstract}
RESUMO
Treinamento mental é uma forma de simulação. O artigo tem objetivo de analisar, na literatura, os efeitos do treinamento mental na performance de tenistas. Utilizou-se as bases de dados LILACS, MEDLINE e SciELO. Para a obtenção dos estudos foram usados os descritores de assunto: tênis; atenção e imaginação. Foram incluídos estudos empíricos e revisões da literatura em língua portuguesa e inglesa. A partir da pesquisa efetuada encontrou-se um total de 2408 artigos. Ao final da seleção 9 estudos atendiam os critérios aplicados. Os estudos revisados apontam que o treinamento mental tem um efeito importante no desempenho dos tenistas.
\end{abstract}

PALAVRAS-CHAVE: Tênis; treinamento mental; treinamento da imaginação; atenção. 
Treinamento mental é uma forma de simulação. É semelhante a uma experiência sensorial real, mas toda a experiência ocorre na mente (WEINBERG; GOULD, 200 I).

O tênis é um esporte que muito tem a ver com o desenvolvimento físico e mental daqueles que o praticam. Envolve movimentos físicos de vários outros esportes, como correr, saltar e arremessar. Para tanto, o tênis pressupõe atributos motores básicos de força, rapidez, resistência e destreza (SHARP, 1979).

Antigamente, para muitos tenistas não existia uma programação de treinamentos, não havia preocupação com os aspectos físicos e mentais dos jogadores. Os treinos se baseavam em amistosos, com ênfase na técnica e tática do jogo (FARIA, 2002).

Enquanto esporte individual, o tênis sugere demandas psicológicas específicas, e a maioria está relacionada com a capacidade do jogador em saber lidar com seus próprios erros.

Ao realizar um treinamento mental com objetivo de evitar-se o bloqueio mental durante jogos de tênis, o tenista estará apto a desenvolver melhor a sua potencialidade competitiva (GIFFONI, 1989). A utilização do treinamento mental valoriza o domínio da atenção e concentração mais do que outras habilidades, que busca uma base sólida para a autoconfiança (GALLWEY, 1996).

A natureza da tarefa e o nível de habilidade do realizador afetam a forma como o treinamento mental melhorará o desempenho do tenista. Atletas iniciantes e atletas altamente qualificados que usam o treinamento mental em tarefas cognitivas apresentam efeitos mais positivos (WEINBERG; GOULD, 200 I).

Praticar treinamento mental é explorar o ilimitado potencial que existe dentro de cada tenista. Este tipo de treinamento pode ser realizado através da atenção no tênis e também através do treinamento da imaginação.

A atenção pode ser considerada mais amplamente como a concentração de esforço mental sobre eventos sensoriais ou mentais (SOLSO, 1995). É observada (atenção) como uma habilidade de grande importância na hora de desempenhar a atividade esportiva do tênis de campo (ABES, 1999).

Tudo o que precisamos ao começar a praticar a atenção no tênis é de um objeto apropriado no qual focalizar a atenção. No tênis, o objeto mais conveniente e prático é a própria bola. A ordem repetida com maior frequência numa quadra provavelmente é: observe a bola. No entanto, poucos jogadores a vêem bem (GALLWEY, 1996).

Já o treinamento da imaginação é uma técnica mental que programa a mente e o corpo a responder de forma correta a um movimento almejado (LANG, 1980). A imaginação permite recriar e criar experiências no plano imaginário (WILLIAMS, 1994). 
A imaginação ou treinamento da imaginação pode ajudar na melhoria de habilidades. Ao tentar melhorar um "serviço" no tênis, por exemplo, pode-se assistir um jogador profissional e então mentalmente reproduzir suas ações, observando-o para melhorar sucesso esportivo. Ou pode-se reproduzir e observar o próprio serviço, ajustando a informação armazenada que guia a performance física da ação (WRAGA; KOSSLYN, 2003).

O treinamento da imaginação recebe muita atenção por seu papel no desempenho atlético e é frequentemente incluído no treino de habilidades psicológicas dirigido aos atletas para complementar seus programas de treinos (BEAUCHAMP, 1996).

Nesse contexto, o presente estudo tem como objetivo analisar os efeitos do treinamento mental na performance de atletas de tênis de campo, com base em uma revisão sistemática da literatura.

\section{MÉTODO}

A pesquisa caracteriza-se como uma revisão sistemática sobre estudos, analisando os efeitos do treinamento mental no desempenho de atletas de tênis de campo. Os critérios adotados para a seleção de artigos foram os publicados entre os anos de 2000 a 2009 (caracterizando assim esta pesquisa como uma revisão sistemática referente aos artigos publicados nesta última década).

A revisão da literatura foi efetuada no período de janeiro a maio de 2010 utilizando-se as bases de dados LILACS (Literatura Latino-Americana e do Caribe em Ciências da Saúde), MEDLINE (Literatura Internacional em Ciências da Saúde) e SciELO (Scientific Electronic Library Online), selecionadas por apresentarem produções científicas vinculadas à área das ciências da saúde em geral. Também foram analisadas as listas de referências dos artigos identificados. Para a obtenção dos estudos foram utilizados como descritores de assunto, nos campos "palavras", "descritor de assunto", "palavras do título", "título" e "resumo", as seguintes palavras-chave: tênis; tênis e atenção; tênis e imaginação; tennis; tennis e attention; tennis e imagination.

Foram incluídos estudos empíricos e revisões de literatura na língua portuguesa e inglesa indexados nas bases de dados. Não foram selecionados estudos como teses, dissertações, monografias, comentários e resenhas. Optou-se por não incluir teses, dissertações e monografias, comentários e resenhas, visto que a realização de uma busca sistemática das mesmas é inviável logisticamente.

Foram selecionados inicialmente 2408 artigos, dos quais, a partir da leitura de seus títulos, restaram 479. Estes 1929 estudos foram excluídos por não apresentarem, em seus títulos, os descritores de assunto propostos. Em seguida, por não constar em seus resumos a utilização de técnicas do treinamento mental como alternativa de melhoria na performance de tenistas, 470 estudos foram excluídos. 
Desta maneira, 09 estudos foram selecionados para análise final, subdivididos nas categorias atenção no tênis (03 artigos) e treinamento da imaginação (06 artigos).

\section{RESULTADOS E DISCUSSÃO}

A partir da pesquisa efetuada nas bases de dados descritas, encontrou-se um total de 2408 referências relacionadas ao tema. No entanto, ao final da seleção 09 estudos respondiam aos critérios aplicados (Figura 0I).

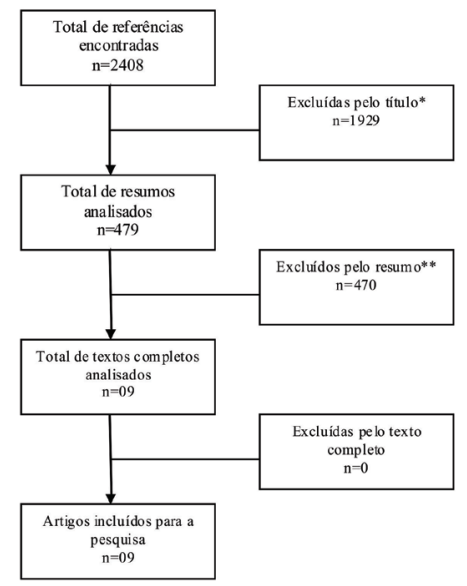

Figura 0I - Sumário de inclusão e exclusão dos estudos que relacionam o treinamento mental no tênis e os artigos selecionados para a pesquisa.

* Não apresentavam, em seus títulos, os descritores de assunto propostos; ** Não constavam em seus resumos a utilização de técnicas do treinamento mental como alternativa de melhoria na performance de tenistas.

Para melhor discussão dos resultados da revisão sistemática, dividiu-se em duas categorias: a) atenção no tênis; b) treinamento da imaginação.

\section{ATENÇÃO NO TÊNIS}

Em relação à atenção no tênis como ferramenta auxiliar para o treinamento mental de tenistas, selecionamos 3 estudos para discussão.

Dias e Teixeira (2006) descrevem uma intervenção psicológica durante quatro meses realizada junto a uma equipe infanto-juvenil de tênis, pertencente ao departamento de tênis de um clube social em uma cidade do interior do Rio Grande do Sul. Na época da intervenção o clube contava com cerca de 200 atletas tenistas, tendo como foco crianças, adolescentes tenistas e equipe de treinadores. 
Foram relatadas intervenções em grupos e individuais junto aos atletas, e conversas com os treinadores e pais. Após 4 meses, os treinadores relataram a importância de trabalhar aspectos relacionados à psicologia do esporte e à atenção no tênis, e os atletas demonstraram interesse em continuar com os trabalhos individuais e em grupo. Foi concluído que os exercícios do treinamento mental e da psicologia do esporte, associados à atenção e concentração no tênis, só favoreceram no controle da ansiedade que os atletas apresentavam em quadra.

Mamassis e Doganis (2004) relatam o impacto de temporada de um programa de treinamento mental, em dois grupos de tenistas de elite juniores. Um grupo de tenistas ( $n=5$ ), em adição a sua prática esportiva usual de tênis, foi exposto a cinco diferentes habilidades psicológicas: definição de objetivos, pensamento positivo e auto-fala, concentração e rotinas, técnicas de regulação da excitação, e imagens. E já o outro grupo de elite de jogadores de tênis juniores $(n=4)$, seguiu a mesma quantidade e qualidade da prática de tênis, mas não recebeu nenhuma prática de treinamento mental. Os resultados indicaram um aumento na dimensão do sentido da ansiedade somática, ansiedade cognitiva e autoconfiança para o grupo de intervenção no pós-teste. Foi concluído que, após o treinamento mental, a eficácia dos exercícios de atenção no tênis e na eliminação de problemas específicos de desempenho destes tenistas.

Cerin et al. (2000) investigaram a ansiedade pré-competitiva e os aspectos emocionais de atletas tenistas, onde a descrição dos complexos estados emocionais deveria assumir duas linhas paralelas. A primeira avaliaria o estado e o acervo emocional do tenista, e já a segunda linha iria examinar o conjunto de emoções básicas de todos envolvidos no ambiente, e concentrar-se nas diferenças individuais e fatores que determinam essas diferenças. Pode-se perceber que o treinamento de atenção no tênis só favorecerá a diminuir essa ansiedade pré-jogo.

Duas pesquisas foram escritas na língua inglesa, e ocorreram na Inglaterra e Grécia. Já na língua portuguesa a pesquisa foi realizada no Brasil.

Os três estudos foram publicados entre os anos de 2000 e 2006, com o seu número amostral variando entre 4 e 200 participantes. Com sessões de treinamento mental num período de até 4 meses, estes estudos analisaram a atenção no tênis e os efeitos do treinamento mental na performance de atletas de tênis de campo.

Somando-se os números amostrais, constata-se que 209 indivíduos foram avaliados nestes estudos. Segundo informações colhidas nestas publicações, todos os participantes são do sexo masculino. 
Em relação ao treinamento da imaginação como mecanismo promotor de treinamento mental para tenistas, selecionamos 6 estudos para discussão.

Passos et al. (2006) estudaram dois grupos de atletas, tenistas e praticantes de rugby. $\bigcirc$ estudo tinha como objetivo determinar a existência de diferenças na evolução do desempenho das seguintes tarefas: a placagem no Rugby e o primeiro serviço no Tênis. Neste estudo participaram 10 jogadores de tênis ( $n=10$; cinco para cada abordagem: ecológica e cognitivista) dos escalões iniciados, infantis e cadetes, estando a faixa etária compreendida entre 10 e 14 anos de idade. Os treinos realizavam-se três vezes por semana, e cada sessão possuía 90 minutos de duração. $\bigcirc$ estudo realizou-se durante três semanas, totalizando nove sessões de treino. Em cada sessão de treino cada jogador realizava 3 séries de 10 serviços. Nos resultados, a análise gráfica foi construída com base nos valores de média e desvio padrão de todos os participantes em cada sessão. Foi concluído que ambas abordagens, através do feedback e mentalização realizadas pelos tenistas, possibilitou observar uma evolução no desempenho do primeiro serviço destes tenistas.

Coelho et al. (2008) analisou o efeito da imaginação no desempenho e na precisão do saque no tênis de campo; o estudo objetivou investigar como uma intervenção com treinamento mental (imaginação) afetaria o desempenho de jogadores de tênis juniores brasileiros em duas situações distintas do saque no tênis de campo: ( I ) saque sem precisão e (2) saque com precisão. Os dados foram coletados de uma amostragem de 46 sujeitos, jogadores de tênis de campo, do sexo masculino, com idade entre 14 a 16 anos e média de 15,06 0 0, 83 anos, registrados na Federação Paranaense de Tênis. Os sujeitos foram divididos em dois grupos: Grupo controle (GI), 22 sujeitos que participaram apenas do treinamento de aprimoramento da técnica do saque, e Grupo Experimental (G2), composto por 24 sujeitos, os quais participaram do treinamento da técnica e aplicação da intervenção da imaginação. Os resultados demonstraram melhora do grupo experimental no desempenho do saque na situação saque sem precisão, após a intervenção da imaginação. Já na situação de precisão, o resultado não foi significativo, demonstrando a efetividade da intervenção com treinamento mental (imaginação) na autoconfiança somente na situação que não requereu ação com precisão motora. Conclui-se que a imaginação é um método eficiente para melhoria do desempenho no saque do tênis de campo.

Gammage et al. (2000) investigou que a imaginação é uma peça importante das funções cognitivas e motivacionais em muitas áreas da vida, incluindo o esporte. $\bigcirc$ objetivo do estudo foi analisar como as funções cognitivas e motivacionais do exercício de imagens mentais variam com o sexo, a frequência do exercício e tipo 
de atividade. Os participantes $(n=577)$ completaram o exercício Imagery Questionnaire, que mede a aparência, a energia de imagens técnicas. Os resultados do estudo apontaram que homens usaram imagens muito mais técnicas do que as mulheres, enquanto mulheres usavam imagens de aparência significativamente maiores do que os homens. Conclui-se que a imaginação é muito recomendada para a utilização em atividades esportivas.

Taylor e Shaw (2002) analisaram o efeito do treinamento da imaginação na modalidade esportiva do golfe. $O$ objetivo deste estudo foi determinar os efeitos das imagens de resultados positivos e negativos sobre o desempenho do "putting" do golfe. De acordo com Pelz (2000), o "putt" do golfe é uma habilidade motora que envolve uma série de variáveis que têm uma influência decisiva na sua aprendizagem, nomeadamente a concentração, a postura corporal, a rotina, a experiência e a amplitude do movimento. Mas no estudo de Taylor e Shaw (2002), os jogadores de altas e baixas capacidades executaram a tarefa do golfe do "putting" utilizando imagens em três condições: (a) a condição de imagem positiva de resultados, (b) a condição de imagem negativa do resultado e (c) uma condição de controle não imaginário. Descobriu-se que a imagem de resultado negativo foi prejudicial para o "putting" e seu desempenho, porém o desempenho, na condição de resultados positivos da imagem não era melhor que o desempenho na condição de controle. Há também evidências que sugerem que imagens de resultado negativo foram prejudiciais para a confiança e a performance. Os resultados deste estudo sugerem que os jogadores devem evitar a visualização de imagens negativas, pois isso pode danificar tanto a confiança como o desempenho. Essas conclusões podem também ser projetadas para o tênis de campo.

Coelho et al. (2007) avaliou o uso de imagens de intervenção no desempenho das duas habilidades motoras do tênis (habilidades aberta ou fechada no tênis de campo), no pré e pós-teste em um grupo controle. $\bigcirc$ saque ou serviço na modalidade esportiva tênis e a recepção de saques ou serviços foram selecionados como elementos representativos de habilidades abertas e fechadas, respectivamente. Foram avaliados 48 indivíduos, tenistas do sexo masculino, com idades 16-18 anos $(M=17,2)$, que foram divididos em dois grupos: $(1)$ o de prática técnica, que foi utilizado apenas como grupo controle e (2) Grupo Imaginário, que receberam as duas imagens e práticas técnicas. Achados sugerem que os resultados de imagem positiva podem ser mais poderosos para melhorar o desempenho dos movimentos de habilidades fechadas em comparação aos movimentos de habilidades abertos.

Beilcock et al. (200l) analisou o impacto das imagens de supressão, a frequência dessa supressão, e as tentativas para substituir imagens negativas de erro, montando imagens positivas no "putting" para melhorar o desempenho dos golfistas. 
Foram avaliados golfistas iniciantes $(n=126)$, e atribuíram a este grupo controle a ausência de imagens, e já ao outro grupo, o experimental, com outro tipo de imagens (positivas, de supressão, e com substituição de supressão). Os resultados mostraram que o grupo de imagens positivas havia melhorado seu desempenho. Estes resultados sugerem que as aplicações frequentes de imagens supressivas prejudicam o desempenho e que a tentativa de substituir imagens negativas com os corretivos não irão diminuir os danos na execução do "putting" quando executado com imagens negativas.

A maioria das pesquisas $(n=4)$ escritas na língua inglesa foi realizada em quatro países, de dois continentes, destacando-se o Canadá, Inglaterra, Brasil e Estados Unidos, com respectivamente uma publicação de cada país.

Os seis estudos foram publicados entre os anos de 2000 e 2008, com número amostral variando entre 10 e 577 participantes. Com sessões durando até 90 minutos, realizadas até 3 vezes por semana num período de até 3 semanas, estes estudos analisaram o treinamento da imaginação e os efeitos do treinamento mental na performance de atletas de tênis de campo.

Somando-se os números amostrais, constata-se que 807 indivíduos foram avaliados nestes estudos. Segundo informações colhidas nestas publicações, a maioria dos participantes (100\%) é do sexo masculino.

\section{CONCLUSÃO}

O treinamento da imaginação aparece na literatura com um volume maior de trabalhos publicados, sendo considerado um ótimo meio de exercitar o treinamento mental, e com isso favorece o desempenho de tenistas na prática de sua modalidade esportiva. É destacada a melhoria que o treinamento da imaginação pode promover na realização do saque ou serviço no tênis.

A mentalização também funciona por meio do desenvolvimento e do refinamento de habilidades psicológicas, e a mentalização pode melhorar a concentração, reduzir a ansiedade e aumentar a confiança, importantes habilidades psicológicas para melhorar a performance. $\bigcirc$ treinamento da imaginação é um instrumento conveniente e efetivo para prática do tênis de campo, podendo ser eficaz tanto antes quanto durante uma competição de tênis.

Já a relação entre treinamento mental e atenção no tênis mostra que, quando o tenista atingir um bom nível de atenção, ele está realmente dentro do jogo, formando um todo com a raquete, a bola e a batida, o atleta descobre seu verdadeiro potencial. E atenção no tênis auxilia os tenistas no controle da ansiedade que apresentam antes da partida ou em quadra. 
Os estudos revisados apontam que o treinamento mental tem um efeito muito importante no desempenho esportivo de tenistas. Sugerem-se mais estudos com o objetivo de ampliar a discussão sobre esse tema.

\section{Mental Training in Tennis: a Systematic Review of Literature}

ABSTRACT: Mental training is a form of simulation. This article aimed to review the literature about the effects of mental training in the performance of tennis players. We used databases LILACS, MEDLINE and SciELO. To obtain the studies it was used the subject descriptors: tennis; attention and imagination. We included empirical studies and literature reviews in English and Portuguese. From the survey conducted it was found a total of 2408 articles. At the end 9 studies met the selection criteria applied. The reviewed studies indicate that mental training has an important effect on the performance of tennis players KEYWORDS: Tennis; Mental Training; Training of the Imagination; Attention.

\section{Tenis en formación mental: una revisión sistemática de publicaciones}

RESUMEN: El entrenamiento mental es una forma de simulación. El artículo tuvo como objetivo revisar en la literatura los efectos del entrenamiento mental en el rendimiento de los jugadores de tenis. Se utilizó la base de datos LILACS, MEDLINE y SciELO. Para obtener los estudios utilizó los descriptores de asunto: tenis, atención y imaginación. Se incluyeron los estudios empíricos y revisiones de la literatura en Inglés y Portugués. De la encuesta realizada encontró un total de 2408 artículos. Al final 9 estudios cumplieron los criterios de selección. Los estudios revisados indican que el entrenamiento mental tiene un efecto importante sobre el rendimiento.

PALABRAS CLAVE: Tênis; entrenamiento mental; la formación de la imaginación; atención.

\section{REFERÊNCIAS}

ABES, L. O. A ansiedade em tenistas de alto nível durante o jogo. Trabalho de Conclusão de Curso (Educação Física), Centro de Desportos, Universidade Federal de Santa Catarina, Florianópolis, 1999.

BEAUCHAMP, P. H. et. al. Effects of cognitive-behavioral psychological skills training on the motivation, preparation and putting performance of novice golfers. The Sport Psychologist, vol. 10, p. 157-170, 1996.

BEILCOCK, S. L. at al. Don't miss: The debilitating effects of suppressive imagery on golf putting performance. J Sport Exerc Psychol, vol. 23, p. 200-221, 2001.

CERIN, E. et al. Temporal patterning of competitive emotions: critical review. J Sports Sci, vol. 18, p. 605-626, 2000.

COELHO, R.W. et al. Imagery intervention in open and closed tennis skill performance. Percept Mot Skills, vol. I05, p. 458-468, 2007. 
COELHO, R.W. et al. O efeito da imaginação no desempenho e na precisão do saque no tênis de campo. Rev. Bras. Cineantropom. Desempenho hum., Florianópolis, vol.10, n. 2, p. 176-183, abr./jun. 2008.

DIAS, M. H.; TEIXEIRA, M. A. P. - Psicologia do esporte e jovens tenistas: relato de uma experiência. Psico., Porto Alegre; vol. 37, n. 2, p. 191-198, maio/ago. 2006.

FARIA, E. Tênis \& Saúde: guia básico de condicionamento físico. Barueri: MANOLE, 2002.

GALLWEY, W. T. O jogo interior de tênis. São Paulo: Textonovo, 1996.

GAMMAGE, K. L.; HALL, C. R.; RODGERS, W. M. More about exercise imagery. Sport Psychol, vol. I4, p. 348-359, 2000.

GIFFONI, E. Tênis: catarse moderna. Porto Alegre: FEPLAM, 1989.

LANG, P. J. et al. Emotional imagery. Conceptual structure and pattern of somato viceral response. Psychophysiology, vol. 17, p. 179-192, 1980.

MAMASSIS, G.; DOGANIS, G. The effects of mental training program on juniors precompetitive anxiety, self-confidence and tennis performance. J Appl Sport Psychol, vol. I6, p. I |8-137, 2004.

PASSOS, P.; BATALAU R.; GONCALVES, P. Comparação entre as abordagens ecológica e cognitivista para o treino da tomada de decisão no Ténis e no Rugby. Rev. Port. Cien. Desp., Porto, vol.6, n. 3, p.305-317, out. 2006.

PELZ, D. Putting bible: the complete guide to mastering the green. New York: Doubleday, 2000.

SHARP, M. G. Tênis: golpes básicos. Rio de Janeiro: Ao Livro Técnico, 1979.

SOLSO, R.L. Cognitive psychology. 4th ed. Boston: Allyn \& Bacon, 1995.

TAYLOR, J. A.; SHAW, D. F. The effect of outcome imagery on golf putting performance. J Sports Sci, vol. 20, p. 607-613, 2002.

WEINBERG, R. S.; GOULD, D. Fundamentos da psicologia do esporte e do exercício. Porto Alegre: Artmed, 200I.

WILLIAMS, J. M. Applied Sport Psychology: Person Growth to Peak Performance. Mayfield Publishing Company, California. 3rd edition, 1994.; I0(2): I76- I83..

WRAGA, M.; KOSSLYN, S. M. Encyclopedia Cognitive Science. Imagery. Psychology Research Library, Harvard University, 2003. (vol. 2).

Recebido em: 29 set. 2010 Aprovado em: 26 jun. 2011

Endereço para correspondência: Jonas Godtsfriedt Rua Maria Manchein de Souza, 210, apto. 703

Bairro Kobrasol

São José - SC

CEP: 88. I02-500 